\title{
Resistance to the destruction of concrete in constructions of height buildings at dynamic loads
}

\author{
Mikhail Berlinov ${ }^{1}$ Marina Berlinova ${ }^{1 *}$, and Alexandr Tvorogov ${ }^{1}$ \\ ${ }^{1}$ Moscow State University of Civil Engineering, Yaroslavskoe shosse, 26, Moscow, 129337, Russia
}

\begin{abstract}
The analysis of the criterion of strength of concrete in structures of high-rise buildings under vibration and shock impacts is presented. The idea of an energy approach to ensuring the strength of concrete and the durability of building structures from reinforced concrete under the influence of shock impacts on the life of such structures is presented in a high-rise construction. A method for determining the strength and durability of concrete in load-bearing building structures made of reinforced concrete for irreversible thermodynamic processes has been developed. Dependences that determine the behavior of concrete in reinforced concrete structures of high stores on the load-bearing structures of a building under the influence of damped oscillations from the operation of air transport on the landing site are determined, taking into account the impact arising from its landing.
\end{abstract}

\section{Introduction}

Today, effective influence on the structure formation of concrete is a feature of new technologies for making structures. As the investigations of various authors [2, 7] show, all known strength theories are phenomenologically based on experimental data. Determination of the criteria for the service life of concrete in building structures [4] is based on the limiting state and the beginning of the process of material destruction [5]. The energy theory of strength for concrete is acceptable with a slightly different formulation, namely: destruction occurs when the energy necessary for its destruction reaches its limiting value [6].

In the scientific and technical literature [1], the problem of stable and unstable force resistance of concrete was demolished depending on the level of the stressed state under hysteresis energy dissipation of deformed reinforced concrete elements. Within the framework of the energy approach, a method of compliance was used, which determines the intensity of energy release during the experimental evaluation of the development of cracks in concrete under the condition of dynamic load.

A stable thermodynamic equilibrium state is an infinitesimal small effect and causes an infinitesimal change in the system, but if an infinitesimal effect can cause a finite change in the thermodynamic state of the system, then it is in an unstable equilibrium state.

\footnotetext{
* Corresponding author: marina.tvor@mail.ru
} 
All equilibrium processes are thermodynamically reversible, which without external influence returns the system to its original state, which cannot be said of thermodynamically irreversible processes. So, especially for concrete, deformation is thermodynamically irreversible process.

\section{Results section}

According to the first law of thermodynamics, the transition of the system to a certain final state "2" from the initial "1", the sum of the work W, the heat Q, and the energy of mass transfer $\mathrm{Z}$ does not depend on the manner in which the transition occurs. The character of the internal state of the system determines the value of the internal energy of the system $U$, determined by the difference in the values of these states

$$
\mathrm{U} 2-\mathrm{U} 1=\mathrm{A}+\mathrm{Q}+\mathrm{Z}
$$

For the case of thermodynamically reversible deformation processes, $Z=0$, this parameter (the work of inertial forces) can be neglected. Then the elementary work done over the system

$$
\delta A=\sum_{k=1}^{n} X_{k} \delta x_{k},
$$

here $\mathrm{X} 1, \ldots, \mathrm{Xn}$ are generalized coordinates for the system of external forces. The work done by these forces (for example, from zero to some final state values) will be equal to

$$
A=\int_{0}^{\left(X_{1}, \ldots X_{n}\right)} \sum_{k=1}^{n} X_{k} \delta x_{k} \cdot
$$

The curvilinear integral (3) depends on the path of integration, since the elementary work (2) is not a complete differential. Then the recording of the total differential will reflect the increment of the internal energy of the system when work done above it is $\delta \mathrm{A}$ and the heat input $\delta Q$

$$
\mathrm{dU}=\delta \mathrm{A}+\delta \mathrm{Q},
$$

that with reference to the process of deformation of concrete it is possible to write down

$$
\delta \mathrm{A}=\sigma \delta \varepsilon
$$

At an equilibrium transition from a given state to an infinitely close one, the increment is determined in terms of the internal energy density $U$

$$
d U=\frac{\partial U}{\partial \varepsilon} \delta \varepsilon+\frac{\partial U}{\partial T} \delta T
$$

Here, taking account of (4) and (5), the record will have the form

$$
\frac{\partial U}{\partial T} \delta T+\frac{\partial U}{\partial \varepsilon} \delta \varepsilon=\sigma \delta \varepsilon+\delta Q
$$

Proceeding from the above, the total differential is the differential of energy, reflecting its change between two states:

$$
U_{2}-U_{1} \int_{1}^{2} d U
$$

The relationship between stresses, strains and temperature is determined by the equation of state:

$$
\sigma=\sigma(\varepsilon, \mathrm{T}) \text { or } \varepsilon=\varepsilon(\sigma, \mathrm{T})
$$

According to the second law of thermodynamics, it is impossible to extract heat from a source and turn it into work at a constant temperature without making changes in the given system and the surrounding space. Then for reversible (equilibrium) processes the entropy of the system: 


$$
\mathrm{dS}=\delta \mathrm{Q} / \mathrm{T} .
$$

And, accordingly, for irreversible (nonequilibrium) processes

$$
\mathrm{dS}>\delta \mathrm{Q} / \mathrm{T} \text {. }
$$

Having written (7) with allowance for $\mathrm{T}$, we obtain

$$
\frac{\delta Q}{T}=\frac{1}{T} \frac{\partial U}{\partial \varepsilon} \delta \varepsilon+\frac{1}{T} \frac{\partial U}{\partial T} \delta T+\frac{1}{T} \sigma \delta \varepsilon,
$$

here for reversible processes $\delta \mathrm{Q} / \mathrm{T}$ is a total differential:

$$
\left(\frac{\partial U}{\partial \varepsilon}\right)_{T}=\sigma-T\left(\frac{\partial \sigma}{\partial T}\right)_{\varepsilon}
$$

and then the entropy difference for two states of the system for reversible processes will have the form

$$
S_{1}-S_{2}=\int_{1}^{2} \frac{\delta Q}{T}
$$

and for irreversible

$$
S_{1}-S_{2}>\int_{1}^{2} \frac{\delta Q}{T}
$$

Since the parameters of temperature and stress tensor, etc. can be measured by conventional instruments, as in the case of nonequilibrium processes, a natural phenomenological definition is obtained. Accordingly, when developing thermodynamic methods for determining the strength and durability of concrete in building constructions for irreversible processes, it can be assumed:

- for the irreversible processes, the principle of local equilibrium (the second law of thermodynamics) remains valid both for reversible processes (4) and (9);

- thermodynamic functions of coordinates and time do not depend, because in each local volume of a concrete body are functions of the same parameters as in the case of equilibrium;

gradients of velocities, temperatures, stresses, etc. in the system under consideration are sufficiently small;

- in the individual elements of the system, the total change in energy and entropy add additively from the changes in the thermodynamic functions.

As can be seen from the analysis, the space of the thermodynamic state of any system is three-dimensional and is determined by parameters characterizing its internal state, when the two parameters are independent, and the third one can always be calculated through them (1absolute body temperature T, 2-specific body volume V, 3- the specific pressure $\rho$ or the internal energy density $U$, or the entropy density $S$, or the enthalpy density $i$, etc.). The change in internal energy as a function of states "1" and "2" is determined by the difference (U2-U1) of the corresponding values and is completely independent of the nature of the process, then $\mathrm{dU}$ is the total differential

$$
\int_{1}^{2} d U=U_{2}-U_{1} \text { and } \Phi d U=0 .
$$

Considering the processes of deformation of concrete in building structures, along a reversible path, for $\mathrm{V}$ and $\rho$ equivalents are the stresses $\sigma$ and relative deformations $\varepsilon$.

When operating high-rise buildings, the problem of their maintenance arises, including in emergency situations, when emergency evacuation of people on the upper floors is necessary, which makes it expedient to use air transport-helicopters. However, when using them, it becomes necessary to provide in the building special landing areas, which are located on the cover. In this case, it is necessary to take into account the impact of additional shock effects arising from the action of the helicopter. 
The load from the operation of air transport is represented in the form of damped harmonic oscillations.

Damping occurs as a result of damping of the oscillations due to the features of the structure of the concrete, which is mathematically reflected by adding to the oscillation equation the recording of a damped time function [1]: $y=A \cdot e^{-\frac{\delta t}{T}} \cdot \sin (\omega t+\varepsilon)$, where $e^{-\frac{\delta t}{T}}$ is the damped function of time.

One cycle of oscillations can be described using the "loading-unloading" diagram presented in [8] (Fig. 1).

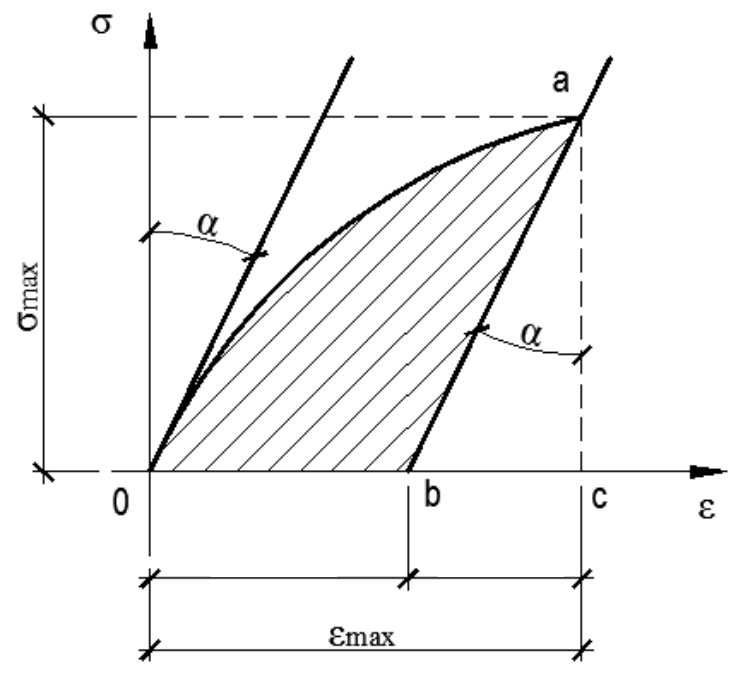

Fig. 1. The diagram "loading-unloading" of a concrete sample: $\varepsilon_{O b .}$ - reversible deformations

when unloading to the instant $\mathrm{t} ; \varepsilon_{\text {H.Ob. }}$ - irreversible deformations; figure $0 \mathrm{ab}$ - hysteresis loop.

In this case, the amplitude of the oscillations will correspond to the maximum stresses in each cycle. It is known that the total deformations correspond to the energy expended for complete deformation in one "loading-unloading" cycle, which can be defined as the area of figure 0ac in Fig. 1:

$$
W=\int_{0}^{\varepsilon_{\max }} \sigma d \varepsilon=\sigma_{\max } \varepsilon_{\max }-\int_{0}^{\sigma_{\max }} \varepsilon d \sigma
$$

Then for $\mathrm{n}$ the number of oscillations, the total deformations can be defined as the sum of the total deformations of each load-unload cycle:

$$
\varepsilon_{\text {full }}=\sum_{i=1}^{n} \int_{0}^{\varepsilon_{\max }} \sigma d \varepsilon=\sum_{i=1}^{n} \sigma_{\max } \varepsilon_{\max }-\int_{0}^{\sigma_{\max }} \varepsilon d \sigma
$$

Thus, with the help of (17), complete deformations from the operation of air transport on the structure of a high-rise building can be determined. The properties of concrete vary from age, as well as from the influence of external influences and the regime of the previous loading.

The influence of impact when landing air transport on the cover-area can be reflected in the form of a diagram of stress-strain state of concrete with preloading at time $t$ (Fig. 2). 


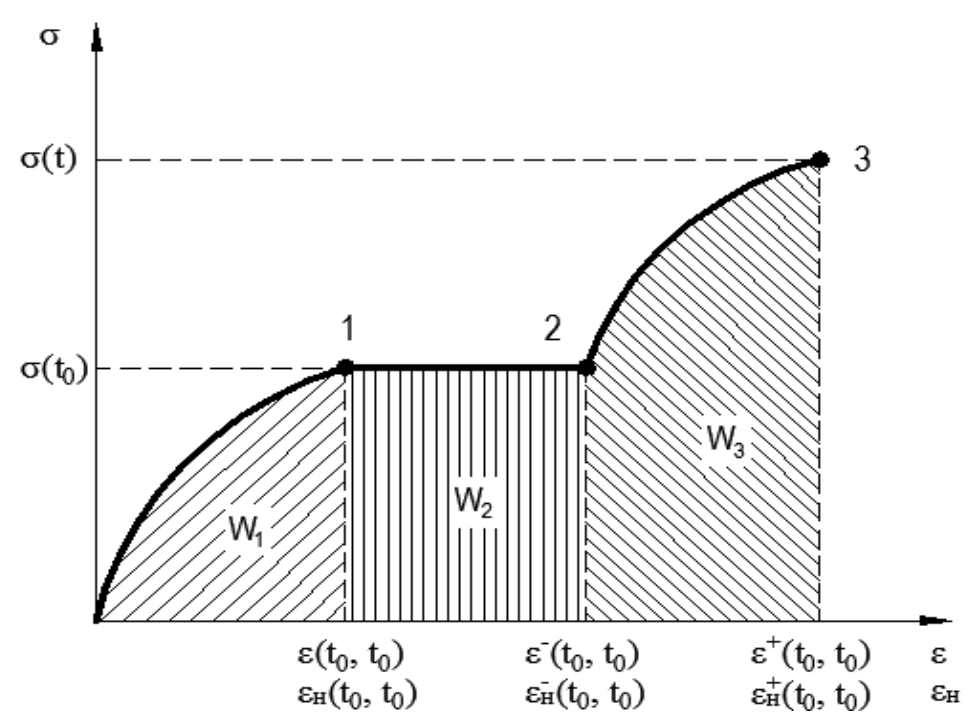

Fig. 2. The diagram of deformation of compressed concrete at its preloading at time $t$.

Here $\sigma\left(t_{0}\right)$ - the stress in the concrete at the time $t_{0} ; \varepsilon\left(t_{0}, t_{0}\right), \varepsilon_{d}\left(t_{0}, t_{0}\right)$ - respectively, the relative and irreversible deformation of concrete under the action of stress; the area of the figure $W_{1}$ - the production of entropy when loaded at a time $t_{0}$; section 1-2 corresponds to simple loading at a stress $\sigma\left(t_{0}\right)$ for a time interval $t_{0}$ from $t$; the area of the figure $W_{2}$ - the increment in the entropy production density over a period of time $t_{0}$ from $t ; t$ - the moment of time, $\sigma(t)$ - the stress at the moment of time $t$, in which the overload occurs; $\varepsilon^{+}\left(t_{0}, t_{0}\right)$, $\varepsilon_{d}^{+}\left(t_{0}, t_{0}\right)$ - respectively, the relative and irreversible deformation of concrete under the action of stress $\sigma(t)$; the area of the figure $W_{3}$ - the increment in the density of entropy production during preloading.

For the process of deformation of real bodies along an irreversible path, in particular concrete, which has the properties of nonlinearity and nonequilibrium, the stresses $\sigma$ and deformation $\varepsilon$ are functionals and cannot simultaneously serve as state parameters, since they strongly depend on deformation.

The total increment in the entropy production density will be equal to the sum of the incremental increments, then at the moment of failure under impact: 


$$
\begin{aligned}
& \Delta S \times T e=\left\{\frac{V_{k}}{E_{b}(t)}\left[\frac{\sigma\left(t_{0}\right)}{R\left(t_{0}\right)}\right]^{m k}+\left\{1-k_{o b . k}+V_{c}\left[\frac{\sigma\left(t_{0}\right)}{R(t)}\right]^{m c}\right\} C^{*}\left(t, t_{0}\right)\right\} \sigma^{2}\left(t_{0}\right)- \\
& \frac{V_{k}}{E_{b}\left(t_{0}\right)} \frac{\sigma^{m k+1}}{R^{m k}\left(t_{0}\right)} d \sigma-\int_{0}^{\sigma\left(t_{0}\right)}\left\{1-k_{o b . c}+V_{c}\left[\frac{\sigma}{R\left(t_{0}\right)}\right]^{m c}\right\} \sigma C^{*}\left(t_{0}, t_{0}\right) d \sigma+ \\
&+\left\{\frac{V_{k}}{E_{b}(t)}\left\{\left[\frac{\sigma(t)}{R(t)}\right]^{m k} \sigma(t)-\left[\frac{\sigma\left(t_{0}\right)}{R(t)}\right]^{m k} \sigma\left(t_{0}\right)\right\}\right\}+\left\{1-k_{o b . k}+V_{c}\left[\frac{\sigma(t)}{R(t)}\right]^{m c}\right\} \sigma(t)- \\
&-\int_{0}^{\sigma\left(t_{0}\right)}\left.\left.-\left\{1-k_{o b . c}+V_{c}\left[\frac{\sigma\left(t_{0}\right)}{R(t)}\right]^{m c}\right\} \sigma\left(t_{0}\right)\right\} C^{*}(t, t)\right\} \sigma(t)- \\
&-\int_{\sigma\left(t_{0}\right)}^{\sigma(t)} \frac{V_{k}}{E_{b}(t)} \frac{\sigma^{m k+1}}{R_{b}^{m k}(t)} d \sigma-\int_{\sigma\left(t_{0}\right)}^{\sigma(t)}\left\{1-k_{o b . c}+V_{c}\left[\frac{\sigma}{R_{b}(t)}\right]^{m c}\right\} \sigma C^{*}(t, t) d \sigma
\end{aligned}
$$

Where $C^{*}(t, t), C^{*}\left(t, t_{0}\right), C^{*}\left(t_{0}, t_{0}\right)$ - a measure of creep; $m$ - the parameter of the nonlinearity of the deformation, depending on the loading regime; $k$ - material constant; $E_{b}(t)$ - instantaneous strain module. On the basis of the analysis given in [8], the derivation of the formula of long-term strength (19) follows, which applicable in the case under consideration:

$$
\begin{aligned}
& \frac{\Delta S T}{R_{d l}(t)}=\frac{V_{k}}{E_{b}(t)}\left[\beta^{m k} \lambda^{2}+\frac{m_{k}+1}{m_{k}+2}\left(1-\lambda^{m k+1}\right)\right]+\left(1-k_{o b . c}+V_{c} \beta^{m c}\right) \lambda^{2} C^{*}\left(t, t_{0}\right)- \\
& -\frac{1}{E_{b}\left(t_{0}\right)} \frac{V_{k}}{m_{k}+2} \beta^{m k} \lambda^{2}-\left(\frac{1-k_{o b . c}}{2}+\frac{V_{c}}{m_{c}+2} \beta^{m c}\right) \lambda^{2} C^{*}\left(t_{0}, t_{0}\right)+\left[\left(1-k_{o b . c}\right) \frac{(1-\lambda)^{2}}{2}+\right. \\
& \left.+V_{c} \eta_{R}^{m c} \frac{m_{c}+1-\lambda^{m c+1}\left(m_{c}+2\right)+\lambda^{m c+2}}{m_{c}+2}\right] C^{*}(t, t)
\end{aligned}
$$

\section{Conclusions}

Thus, it can be seen that on the basis of the assumption introduced in [1] on the significant effect of the dissipation of the force resistance due to the complex influence of the medium and the absorption of the deformation energy, it is possible to determine the influence of vibrational influences on the structure of the building from the operation of air transport when landing on the roof of a high-rise buildings.

Based on the analysis of the second law of thermodynamics with respect to the process of deformation of concrete carried out in [3], and also taking into account the modified record of the first law of thermodynamics with respect to the deformation process, it becomes 
possible to use the entropic strength criterion for analysis of deformation of structures, in particular, from shock effects in the considered the case.

The application of such criteria as the effect of dissipation of force resistance, or entropic criterion of strength in the analysis of deformation of structures of buildings and structures will allow to take into account the real working conditions of structures that will arise during operation, including in extreme situations.

\section{References}

1. V.M. Bondarenko Report on research work on the topic: Fundamentals of applied dissipative theory of survivability of structures. Moscow. 2008.

2. M.V. Berlinov Calculation of the structure of the frame of buildings under dynamic influences from industrial equipment // Industrial and civil construction. 2004. № 6. Pp. 48-49.

3. M.N Berlinova., M.V. Berlinov, A.V. Tvorogov To the question of ensuring the strength of concrete by the method of thermodynamics // Scientific review. 2015 No. 22. Pp. 148 153.

4. M.N. Berlinova, M.V. Berlinov, A.V. Tvorogov Entropy criterion of strength of concrete in building structures // Scientific Review. 2015 No. 22. pp. 162-165.

5. M.V. Berlinov, E.A. Makarenkov Calculation of reinforced concrete structures by the $f$ inite element method taking into account a real description of the actual physical processes // Vestnik MGSU. 2013. № 11. pp. 26-33.

6. M.V. Berlinov, E.A. Makarenkov Criterion of strength of concrete in conditions of a triaxial stress state under dynamic influences // Scientific Review. 2014. No. 7-1. Pp. 197-200.

7. M.N. Berlinova, A.V. Tvorogov The regime strength of concrete in building structures // Natural and technical sciences. 2015. № 6 (84). Pp. 530-532.

8. M.N. Tvorogova Resistance to deformation and destruction of reinforced concrete structures taking into account nonlinear and nonequilibrium properties and loading regimes // Thesis of the candidate of technical sciences. Moscow. 2006. 